\title{
À mots couverts
}

Jean-Marie Privat

\section{(2) OpenEdition}

Journals

Édition électronique

URL : https://journals.openedition.org/clo/2025

DOI : $10.4000 /$ clo.2025

ISSN : 2266-1816

Éditeur

INALCO

\section{Édition imprimée}

Date de publication : 1 janvier 2014

ISBN : 978-2-85831-222-1

ISSN : 0396-891X

\section{Référence électronique}

Jean-Marie Privat, «À mots couverts », Cahiers de littérature orale [En ligne], 75-76 | 2014, mis en ligne le 05 mai 2015, consulté le 01 juillet 2021. URL : http://journals.openedition.org/clo/2025 ; DOI : https://doi.org/10.4000/clo.2025

Ce document a été généré automatiquement le 1 juillet 2021.

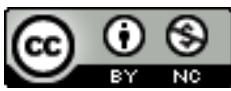

Cahiers de littérature orale est mis à disposition selon les termes de la Licence Creative Commons Attribution - Pas d'Utilisation Commerciale 4.0 International. 


\title{
À mots couverts
}

\author{
Jean-Marie Privat
}

\section{Le dépaysement culturel}

1 Flaubert aurait donc bien fait l'expérience directe et enfantine d'une certaine altérité culturelle du proche. Au demeurant, cette relation vivante au folklore verbal faisait partie de la prime socialisation d'alors (la parole conteuse et fabuleuse des servantes rustiques à domicile est un topos des souvenirs des enfances bourgeoises et urbaines au $\mathrm{XIX}^{\mathrm{e}}$ siècle) ${ }^{2}$. Ce contact premier, privé et privilégié avec des fragments de l'imaginaire rustique s'inscrivait aussi à cette époque dans l'aura romantique et nationale qui entoure la découverte anthropologique des mœurs paysannes et plus généralement du folklore des provinces (Fabre, 2010, 5-75) ${ }^{3}$.

2 C'est dans un même contexte friand d'escapades pittoresques, de curiosités artistiques et de coutumes étranges ou de scènes de mœurs singulières que le jeune Flaubert et son ami Maxime Du Camp courent la Bretagne (mai-août 1847), consignant à tour de rôle leurs observations et leurs impressions dans des carnets, trace d'une aventure culturelle et trame d'un récit de voyage à pied (le plus souvent) dans les fines terrae provinciales ${ }^{4}$. Ce témoignage-là sera publié tardivement sous le titre évocateur de Par les champs et par les grèves (1886). Voici par exemple ce qu'écrivent nos deux compères lors de leur étape à Pont-l'Abbé :

Ce soir même, nous allâmes dans un village des environs, voir l'inauguration d'une aire à battre. Deux joueurs de biniou, montés sur le mur de la cour, poussaient sans discontinuer le souffle criard de leur instrument, au son duquel courait au petit trot, en se suivant à la queue du loup, deux longues files d'hommes et de femmes qui serpentaient et s'entrecroisaient. Les pas lourds battaient le sol, sans souci de la mesure, tandis que les notes aiguës de la musique se précipitaient l'une sur l'autre dans une monotonie glapissante. Ceux qui ne voulaient plus danser s'en allaient, sans que la danse en fût troublée, et ils rentraient de suite quand ils avaient repris haleine. Pendant plus d'une heure que nous considérâmes cet étrange exercice, la foule ne s'arrêta qu'une fois, les musiciens s'étant interrompus pour boire un verre de cidre ; puis les longues lignes s'ébranlèrent de nouveau et se remirent à tourner. À l'entrée de la cour, sur une table, on vendait des noix, à côté était un broc d'eau- 
de-vie, par terre une barrique de cidre; non loin se tenait un particulier en casquette de cuir et redingote verte ; près de lui, un homme en veste avec un sabre suspendu par un baudrier blanc: c'était le commissaire de police de Pont-l'Abbé avec son garde champêtre.

Bientôt, M. le commissaire tira sa montre de sa poche, fit un signe au garde qui alla parler aux paysans, et l'assemblée se dispersa. (Flaubert, 1989, 227-228)5. symboliques à la fois prochains et lointains, singuliers et détonants. Flaubert jeune bachelier avait déjà fait l'expérience par monts et par vaux d'une France exotique, la France des bourgs des villes méridionales cette fois-là. Il se souviendra d'avoir porté «toute une journée un pantalon de paysan et de s'être exercé à aller nu-pieds » (2001, 232), de s'être plu à « réciter tout haut des vers [...] tout seul dans la campagne », la cadence le faisant marcher comme s'il « chantait » (233), ou encore à Marseille de s'être réjoui de voir « le peuple se réjouir » (265):

Aux portes des guinguettes, c'était une foule mouvante, chaude et gaie, qui se dressait sur la pointe des pieds pour voir ceux qui étaient attablés, qui jouaient et qui fumaient. Nous nous y sommes mêlés et à travers les vitres obscurcies nous avons vu, tout au fond d'une grande pièce, la représentation d'un mystère provençal [...]. Le dialogue était vif et animé, improvisé sans doute, plein de saillies à coup sûr à en juger par les éclats de rire et les applaudissements. Tous ces braves gens écoutaient et goûtaient l'air avec respect et recueillement de manière à réjouir un poète s'il passait par là. (Flaubert, 2001, 266).

\section{Une familiarité distante}

4 Le romancier des «mœurs de province » témoigne de cette familiarité distante avec la culture paysanne ou la vie populaire et anonyme - par personnages interposés :

L'air du bal était lourd; les lampes pâlissaient. On refluait dans la salle de billard. Un domestique monta sur une chaise et cassa deux vitres; au bruit des éclats de verre, madame Bovary tourna la tête et aperçut dans le jardin, contre les carreaux, des faces de paysans qui regardaient. Alors le souvenir des Bertaux lui arriva. Elle revit la ferme, la mare bourbeuse, son père en blouse sous les pommiers, et elle se revit elle-même, comme autrefois, écrémant avec son doigt les terrines de lait dans la laiterie. Mais, aux fulgurations de l'heure présente, sa vie passée, si nette jusqu'alors, s'évanouissait tout entière [...]. Elle était là ; puis autour du bal, il n'y avait plus que de l'ombre, étalée sur tout le reste. (Flaubert, 1971, 53).

5 Flaubert ne s'interdit pas non plus d'insérer dans l'économie discursive de son roman des fragments de cosmologie rurale (ou donnés comme tels):

Un matin, le père Rouault vint apporter à Charles le payement de sa jambe remise : soixante et quinze francs en pièces de quarante sous et une dinde. Il avait appris son malheur, et l'en consola tant qu'il put. - Je sais ce que c'est! disait-il en lui frappant sur l'épaule; j'ai été comme vous, moi aussi ! Quand j'ai eu perdu ma pauvre défunte, j'allais dans les champs pour être tout seul ; je tombais au pied d'un arbre, je pleurais, j'appelais le Bon Dieu, je lui disais des sottises ; j'aurais voulu être comme les taupes, que je voyais aux branches, qui avaient des vers leur grouillant dans le ventre, crevé, enfin. Et quand je pensais que d'autres, à ce moment-là, étaient avec leurs bonnes petites femmes à les tenir embrassées contre eux, je tapais de grands coups par terre avec mon bâton; j'étais quasiment fou, que je ne mangeais plus; l'idée d'aller seulement au café me dégoûtait, vous ne croiriez pas. Eh bien, tout doucement, un jour chassant l'autre, un printemps sur un hiver et un automne par-dessus un été, ça a coulé brin à brin, miette à miette ; ça s'en est allé, 
c'est parti, c'est descendu, je veux dire, car il vous reste toujours quelque chose au fond, comme qui dirait... un poids, là, sur la poitrine ! (1971, 21-22).

6 Cette attention " réaliste » aux différenciations sociales et aux variations culturelles était (beaucoup) plus manifeste encore dans les brouillons de Madame Bovary, par exemple lorsque le romancier s'essayait à une esquisse ethnographique des enfances villageoises du jeune Charles :

Jusqu'à quinze ans, il vécut dans la liberté oisive des garçons de la campagne levés dès le soleil et sortis toute la journée pour aller dénicher des merles et qui ne rentrent que pour souper sans qu'on sache où ils sont (...). Il suivait les laboureurs aux champs et poursuivait à coups de motte de terre dans les sillons les corbeaux qui s'envolaient. Il mangeait des mûres le long des fossés, gardait les dindons avec une gaule, fanait à la moisson et allait dans les bois pour se couper des baguettes ou s'arranger avec son couteau des sifflets d'osier. Il savait brasser le cidre, tresser des caches aux fouets et bien ruer des pierres. Ils partaient à plusieurs pêcher au bout d'un roseau des grenouilles dans les étangs. Il effarouchait les dindons dans la haie, poursuivait les rats dans la grange et dans les près fauchés les reinettes. Sous la remise, il se balançait aux brancards des charrettes. Il s'amusait à rouler sur les tas d'herbe, rentrant déchiré. L'hiver dans la grange, il s'amusait tout seul, à se laisser glisser du haut en bas des bottes de pailles. Il jouait à la marelle les jours de pluie avec des gamins du pays sous le porche de l'église, et aux grandes fêtes, suppliait le bedeau de lui laisser sonner les cloches, pour se pendre de tout son corps à la grande corde et se sentir emporté par elle dans sa volée ${ }^{6}$.

7 Le peuple serait-il « à la mode » chez les écrivains (Michelet, 1974, 151) ${ }^{7}$, en ce milieu du XIX ${ }^{\mathrm{e}}$ siècle? Sans doute - mais chez Flaubert il s'agit moins en définitive d'une ethnographie des mœurs et des coutumes populaires ${ }^{8}$ que de la compréhension anthropologique des structures symboliques d'une pensée sauvage indigène. Le récit de Flaubert textualise en effet des modes de pensée magico-folklorique que la narration prend en charge dans sa dynamique propre (et pour ses fins propres). Nous examinerons ici plus particulièrement quelques modalités d'appropriation sémiostylistique des signes et des paroles du malheur.

\section{Les mauvais signes}

8 La richesse anthropologique (et historique) du roman tient moins à ses effets de réel ethnologiques qu'à la sémiotisation narrative de systèmes de créance populaires et folkloriques. L'univers des signes oraculaires et des pratiques "superstitieuses » est certes présent dans la thématique de Madame Bovary, mais à titre sociologique et à la charge des personnages seuls (non sans ironie d'ailleurs de la part du narrateur). En voici deux exemples, divination galante d'un amoureux de circonstance ou discours positiviste du pharmacien Homais :

Aujourd'hui, j'ai le bonheur d'être avec vous... Emma rougit. Il (Rodolphe) n'acheva point sa phrase. Alors il parla du beau temps et du plaisir de marcher sur l'herbe. Quelques marguerites étaient repoussées. - Voici de gentilles pâquerettes, dit-il, et de quoi fournir bien des oracles à toutes les amoureuses du pays. Il ajouta : - Si j'en cueillais. Qu'en pensez-vous? - Est-ce que vous êtes amoureux? fit-elle en toussant un peu. - Eh ! eh ! qui sait ? répondit Rodolphe. $(1971,140)$.

$\mathrm{Ah}$ ! vous trouverez bien des préjugés à combattre, monsieur Bovary; bien des entêtements de la routine, où se heurteront quotidiennement tous les efforts de votre science ; car on a recours encore aux neuvaines, aux reliques, au curé, plutôt que de venir naturellement chez le médecin ou chez le pharmacien. $(1971,83)$. 
Flaubert connaissait par ouï-dire direct ${ }^{9}$ ou par culture commune à son époque les croyances folkloriques ordinaires ${ }^{10}$, et les formules proverbiales ou les observations coutumières relatives au malheur et à la mort ne sont pas étrangères à son récit, notamment les prédictions liées au bestiaire domestique (dans des contextes tragiques ou funèbres) :

Mère Rolet, dit-elle en arrivant chez la nourrice, j'étouffe! délacez-moi [...]. Couchée sur le dos, immobile et les yeux fixes, elle (Emma) contemplait les écaillures de la muraille, deux tisons fumant bout à bout, et une longue araignée qui marchait au-dessus de sa tête dans la fente de la poutrelle [...]. Quelle heure est-il ? demanda-t-elle. La mère Rolet sortit, leva les doigts de sa main droite du côté que le ciel était le plus clair, et rentra lentement en disant : - Trois heures, bientôt. - Ah! merci ! merci ! Car il allait venir. C'était sûr! Il aurait trouvé de l'argent ${ }^{11}$. (1971, 313).

Des aboiements continus se traînaient au loin, quelque part. - Entendez-vous un chien qui hurle? dit le pharmacien. On prétend qu'ils sentent les morts, répondit l'ecclésiastique. C'est comme les abeilles; elles s'envolent de la ruche au décès des personnes. - Homais ne releva pas ces préjugés, car il s'était rendormi. M. Bournisien [...] insensiblement baissa le menton, lâcha son gros livre noir et se mit à ronfler [...]. Et ils ne bougeaient pas plus que le cadavre à côté d'eux qui avait l'air de dormir. $(1971,339)$.

L'art narratif de Flaubert consiste, on le voit, à laisser s'introduire ici et là dans la trame du texte une sorte de halo sémantique et idéologique qui sollicite la coopération interprétative du lecteur et inscrit à l'horizon du récit un monde culturel aussi familier qu'étrange, étrangement familier même pour l'instant. L'astuce stylistique de l'écrivain est de jouer des points de suspension et des silences pour laisser advenir des événements de pensée plus ou moins subliminaux. Le discours du curé Bournisien relance ponctuellement cette irruption possible d'un refoulé doxique (soulignée par les signes d'une altérité graphique et lexicale) ${ }^{12}$ :

Ah! ne m'en parlez pas, madame Bovary! Ce matin même, il a fallu que j'aille dans le Bas-Diauville pour une vache qui avait l'enfle; ils croyaient que c'était un sort. Toutes leurs vaches, je ne sais comment... $(1971,116)$.

11 C'est ainsi que se dessine allusivement sinon l'univers complet de la sorcellerie bocagère (Disegni, 1995, 6-50), à coup sûr le monde des cultures orales traditionnelles et des systèmes interprétatifs hétérodoxes.

\section{Une poétique de l'intersigne}

12 En effet, c'est la structure narrative profonde du récit et donc sa cosmologie culturelle propre, bref son écriture même qui est en partie motivée (entre autres schèmes organisateurs bien sûr) et dynamisée par une coalescence littéralement in-ouïe entre une mantique et une sémantique. Inattendue parce que le lecteur moderne n'est en général ni prêt ni préparé à reconnaître dans certains signes discursifs interstitiels des intersignes fatidiques... ${ }^{13}$ Suivons ici un des fils de ce destin du récit, entre présage et malédiction.

\section{Trois poules noires}

13 Emma (née Rouault) s'est empoisonnée. Elle n'existe plus. L'apothicaire adresse au père Rouault « une lettre rédigée de telle façon qu'il était impossible de savoir à quoi s'en 
tenir" $(1971,341)$. Le bonhomme passe sa blouse, prend son chapeau, accroche un éperon et au petit matin part au grand galop rejoindre sa fille, morte ou encore vive :

Tout le long de la route, le père Rouault, haletant, se dévora d'angoisses [...]. Le jour se leva. Il aperçut trois poules noires qui dormaient dans un arbre; il tressaillit, épouvanté de ce présage. Alors il promit à la Sainte Vierge trois chasubles pour l'église, et qu'il irait pieds nus depuis le cimetière des Bertaux jusqu'à la chapelle de Vassonville. (1971, 341).

Le récit conjugue ici trois cosmologies: une mythologie antique (les poules noires comme Parques de basse-cour), une religion chrétienne populaire et rurale (offrandes votives, pèlerinage sacrificiel local) et surtout une mantique folklorique traditionnelle (oiseaux de mauvais augure). Cette polyphonie culturelle est assumée dans la version définitive du texte ${ }^{14}$ qui de fait donne crédit à la mantique paysanne. Le " présage » dit le vrai : Emma est morte. Or, le lecteur et le narrateur savent que la promesse pieuse du père affolé est vaine, sans objet. Ils sont ainsi placés du point de vue narratif et idéologique comme en surplomb critique par rapport aux illusions de la piété orthodoxe et surtout en connivence, en complicité cognitive avec la culture folklorique $\mathrm{du}$ père Rouault. Cette culture fait spontanément corps («tressaillit ", «épouvanté ») avec le fermier des Bertaux... et avec la culture hétérophonique du récit.

\section{Une malédiction}

L'enchaînement des malheurs et des deuils est largement constitutif du roman ${ }^{15}$ et cet enchaînement lui-même a quelque chose d'inexplicable, comme une fatalité ou plutôt comme une malédiction :

Quand il (le père d'Emma) eut repris connaissance, il tomba tout en pleurs dans les bras de Bovary: - Ma fille! Emma! mon enfant! expliquez-moi... ? Et l'autre (Charles) répondit avec des sanglots: - Je ne sais pas, je ne sais pas! C'est une malédiction! (1971, 342).

Il faut reprendre le fil du destin et revenir à la noce de Charles et d'Emma pour saisir quelle pré-diction (ou pré-scription) de malheur s'accomplit :

C'était sous le hangar de la charretterie que la table était dressée [...]. La mariée avait supplié son père qu'on lui épargnât les plaisanteries d'usage. Cependant, un mareyeur de leurs cousins (qui même avait apporté, comme présent de noces, une paire de soles) commençait à souffler de l'eau avec sa bouche par le trou de la serrure, quand le père Rouault arriva juste à temps pour l'en empêcher, et lui expliqua que la position grave de son gendre ne permettait pas de telles inconvenances. Le cousin, toutefois, céda difficilement à ces raisons. En dedans de lui-même, il accusa le père Rouault d'être fier, et il alla se joindre dans un coin à quatre ou cinq autres des invités qui, ayant eu par hasard plusieurs fois de suite à table les bas morceaux des viandes, trouvaient aussi qu'on les avait mal reçus, chuchotaient sur le compte de leur hôte et souhaitaient sa ruine à mots couverts. (1971, 30-31).

La transgression des lois coutumières de l'échange symbolique et des règles de l'hospitalité et de la réciprocité (dons nuptiaux obligatoires et contre-dons rituels) expose inévitablement à une réaction plus ou moins ouverte et violente des victimes publiquement offensées ou pis humiliées ${ }^{16}$. Mais en quoi et pourquoi ces souhaits de malheurs ont-ils une force performative (ou perlocutoire) réelle dans le roman de Flaubert, une efficace magico-poétique en quelque façon? Il est utile de distinguer sommairement deux plans (les contenus de fiction, la rhétorique du récit), même s'ils 
sont conjoints ou plus exactement se miroitent l'un dans l'autre dans le discours du roman. Entre créance culturelle et crédit narratif en somme.

Dans l'univers cérémoniel de la culture paysanne (où "toute offense est défi ", Bourdieu, 2000, 30), si le code de l'honneur est bafoué, si les règles de la socialité rituelle sont ouvertement méprisées, si les usages coutumiers sont d'autorité refoulés ou mal traités ${ }^{17}$, etc., et si l'affront symbolique est à la fois public et manifeste (le petit peuple est traité comme le bas peuple, voué au bout de table et aux bas morceaux des viandes), alors il est ethno-logique que les derniers des derniers traités comme des chiens cherchent vengeance de l'insulte et de l'outrage ${ }^{18}$. Ils disposent d'une arme : la parole. Cette parole collective et virile, anonyme et ésotérique, sera un (mauvais) sort jeté sur l'à venir de la maison Rouault et sur le mariage d'Emma ${ }^{19}$. Le réalisme ethnographique du texte ${ }^{20}$ rencontre les jeux et enjeux de la narration qui est volontiers prédictive ${ }^{21}$. La dramaturgie d'un récit réaliste consiste en effet à laisser à la sagacité du lecteur des indices en attente de concrétisation fictionnelle (Ginzburg, 2010, 218-294; Kliebenstein, 2004, 87-196), tout comme en un sens le décodage des oralités prédictives présuppose une attention aux signes et à leurs intentions.

Le lecteur diligent aura ainsi en mémoire vive la page qui précède le motif des «mots couverts ", page de présentation sociographique des invités à la noce d'Emma et de Charles, page où se trouve en fait pré-inscrit noir sur blanc que les plus pauvres des invités sont destinés à être mal reçus :

Suivant leur position sociale différente, ils avaient des habits, des redingotes, des vestes, des habits-vestes [...]. Quelques-uns encore (mais ceux-là, bien sûr, devaient dîner au bas bout de la table) portaient des blouses de cérémonie, c'est-à-dire dont le col était rabattu sur les épaules, le dos froncé à petits plis et la taille attachée très bas par une ceinture cousue ${ }^{22}$. $(1971,28)$.

Cette pré-science («bien sûr ») inscrit le récit dans la logique des mondes clos, elle l'arrime aussi à l'univers des sociétés à honneur et à leurs impératifs (Pitt-Rivers, 1997). L'ensauvagement culturel du lecteur consiste à accepter de donner toute sa portée au savoir des anthropologues qui ont fait observer combien dans les cultures orales traditionnelles «l'offense qui consiste à négliger quelqu'un [...] a des conséquences funestes [...] ». Le motif de "la mauvaise fée oubliée au baptême ou au mariage» (Mauss, 1991, 208-209) est même un "thème essentiel du folklore européen ", sans parler des cultures lointaines ou anciennes ${ }^{23}$. Mais la féerie ne sied pas au roman moderne et c'est en vain (et non sans ironie ou humour flaubertien) que le père d'Emma promettra à la Sainte Vierge un contre-don propitiatoire («trois chasubles » pour la sainte messe - trois chasubles «brodées d'or » dans les brouillons). Par-delà un apparent pittoresque rustique et naïf, le parcours du pèlerinage envisagé par le paysan semble viser à inverser le temps puisqu'il imagine partir du cimetière familial (il y a bien un cimetière à la ferme des Bertaux...) pour rejoindre la chapelle de Vassonville. Or Vassonville est un lieu de passage particulièrement symbolique dans le roman, un chronotope sémantiquement ou axiologiquement équivoque en réalité. C'est là où au début du roman Charles, jeune officier de santé bientôt veuf, rencontre un petit guide paysan qui lui indiquera le chemin des Bertaux (et de sa future épouse) ${ }^{24}$; c'est encore à Vassonville qu'au lendemain des noces le père de la mariée dira au revoir (adieu ?) à sa fille pour qui s'ouvre un nouveau destin, à la vie à la mort, avec Charles :

Le père Rouault les fit reconduire dans sa carriole et les accompagna lui-même jusqu'à Vassonville. Là, il embrassa sa fille une dernière fois, mit pied à terre et reprit sa route. Lorsqu'il eut fait cent pas environ, il s'arrêta, et il vit la carriole 
s'éloignant, dont les roues tournaient dans la poussière [...]. Il se rappela ses noces, son temps d'autrefois, la première grossesse de sa femme; il était bien joyeux, lui aussi, le jour qu'il l'avait emmenée de chez son père dans sa maison, quand il la portait en croupe en trottant sur la neige ; car on était aux environs de Noël et la campagne était toute blanche [...]. Comme c'était vieux tout cela! Leur fils, à présent, aurait trente ans! Alors il regarda derrière lui, il n'aperçut rien sur la route. Il se sentit triste comme une maison démeublée [...]; il eut bien envie un moment d'aller faire un tour du côté de l'église. Comme il eut peur, cependant, que cette vue ne le rendît plus triste encore, il s'en revint tout droit chez lui. (1971, 31-32).

21 Vassonville est donc un lieu ambivalent, puisque à la fois lié au bonheur des noces et au malheur des familles. Ainsi, comble de fatalité, cette idée de pèlerinage qui s'inscrit fort bien dans la logique culturelle du paysan est en fait un vœu mort-né si l'on suit le travail de signifiance du texte qui trace l'itinéraire votif d'un cimetière familial à un lieu marqué par une douloureuse voire funeste séparation.

Si l'on tire un ultime fil de la parole de malheur et si l'on accepte de considérer que chez Flaubert le petit peuple peut aussi faire l'histoire (sinon l'Histoire), alors on lira que dans la dynamique sémio-culturelle du récit les «mots couverts ${ }^{25}$, cent cinquante pages plus loin, rencontrent leur efficacité narrative et symbolique attendue.

\section{Un malheur à ma charretterie}

On se souvient que le repas des noces se déroule à la ferme des Rouault, sous le hangar de la «charretterie ». Or, ce que le mauvais sort dit à mots couverts, le récit le réalise à découvert. Chaque année, à date fixe, le père Rouault adresse (sans retour) une lettre accompagnée d'un cadeau ( "un dinde » de la ferme). Il fait comme il est d'usage le point sur la vie quotidienne aux Bertaux :

J'ai eu un malheur à ma charretterie, dont la couverture, une nuit qu'il ventait fort, s'est envolée dans les arbres. La récolte non plus n'a pas été très fameuse. Enfin, je ne sais pas quand j'irai vous voir. Ça m'est tellement difficile de quitter maintenant la maison, depuis que je suis seul, ma pauvre Emma! [...].

Quant à moi, je vais bien, sauf un rhume que j'ai attrapé l'autre jour à la foire d'Yvetot, où j'étais parti pour retenir un berger, ayant mis le mien dehors, par suite de sa trop grande délicatesse de bouche. Comme on est à plaindre avec tous ces brigands-là ! Du reste, c'était aussi un malhonnête [...].

Il me fait deuil de ne pas connaître encore ma bien-aimée petite-fille Berthe Bovary [...].

Adieu, mes chers enfants. $(1857,176)$.

On comprend que la couverture de la charreterie violemment arrachée et jetée dans les arbres par un vent nocturne et funeste, un vent de tous les diables ${ }^{26}$, est comme le signe qu'un mauvais sort s'est bien abattu, tôt ou tard, sur les Bertaux ${ }^{27}$ et par métonymie sur tout ce que ce lieu focal représente dans Madame Bovary ${ }^{28}$. Les nouvelles sont sans exception des nouvelles de destruction des biens et des récoltes, de maladie personnelle et de désordre social, enfin d'isolement, de solitude et de deuil. Ce continuum de malheurs signe bien la «ruine" de la "maison ${ }^{29}$. Et la seule note d'optimisme à laquelle l'épistolier fait allusion - la réussite de Charles et le bonheur du ménage - est en fait un tragique (et ironique) contresens sur la situation réelle des époux Bovary :

J'ai appris d'un colporteur qui, voyageant cet hiver par votre pays, s'est fait arracher une dent, que Bovary travaillait toujours dur [...]. Je lui ai demandé s'il 
t'avait vue, il m'a dit que non, mais qu'il avait vu dans l'écurie deux animaux, d'où je conclus que le métier roule. Tant mieux, mes chers enfants, et que le bon Dieu vous envoie tout le bonheur imaginable. $(1971,176)$.

Le Bon Dieu n'envoie et n'enverra aucun bonheur. Le métier ne roule pas fort en vérité et dans l'écurie il y a le cheval poussif de l'officier de santé et «le cheval de maittre » $(1971,161)$ que Rodolphe « offre » à sa maîtresse - avec la « complaisance » de Charles pour leurs promenades en forêt... Le destin du couple court à la catastrophe, comme on sait. Le mareyeur (le cousin marieur) repassera avec ses compagnons sous les fenêtres d'Emma, avec son charroi de soles et autres poissons frais, chantant la Marjolaine "Que demande le chevalier / Une fille à marier / Mon cœur je lui donnerai » :

La nuit, quand les mareyeurs, dans leurs charrettes, passaient sous ses fenêtres en chantant la Marjolaine, elle s'éveillait; et écoutant le bruit des roues ferrées, qui, à la sortie du pays, s'amortissait vite sur la terre : - Ils y (Paris) seront demain! se disait-elle. Et elle les suivait dans sa pensée, montant et descendant les côtes, traversant les villages, filant sur la grande route à la clarté des étoiles. Au bout d'une distance indéterminée, il se trouvait toujours une place confuse où expirait son rêve. $(1971,59)$.

Un présage de très mauvais augure n'avait-il pas de toute façon présidé à la rencontre inaugurale entre les futurs mariés? Charles arrive aux Bertaux, au petit matin :

L'officier de santé, chemin faisant, comprit aux discours de son guide que M. Rouault devait être un cultivateur des plus aisés. Il s'était cassé la jambe, la veille au soir, en revenant de faire les Rois, chez un voisin. Sa femme était morte depuis deux ans. Il n'avait avec lui que sa demoiselle, qui l'aidait à tenir la maison.

Les ornières devinrent plus profondes. On approchait des Bertaux [...]. Le cheval glissait sur l'herbe mouillée ; Charles se baissait pour passer sous les branches. Les chiens de garde à la niche aboyaient en tirant sur leur chaîne. Quand il entra dans les Bertaux, son cheval eut peur et fit un grand écart. (1971, 14-15).

\section{À mots/maux couverts}

27 La magie de la performance énonciative et parfois dénonciative suppose donc chez le lecteur un travail interprétatif très attentif à l'hétérophonie narrative du récit, aux logiques opératoires des systèmes prédictifs à l'œuvre, au travail de signifiance de la culture du texte enfin, loin de tout légitimisme lettré qui rend cette hypothèse de lecture littéralement et littérairement im-pensable ${ }^{30}$.

Certes, l'hétéroculture du roman semble attester combien l'écrivain (et le lecteur) peuvent «à la fois puiser dans l'indignité culturelle des pratiques dominées le sentiment de leur propre dignité et dignifier en daignant les emprunter les pratiques indignes » (Grignon et Passeron, 1989, 61). Mais ces mêmes cultures subalternes et orales ne servent pas ici de seul substrat folklorique à la peinture des «mœurs de province »; elles participent de l'ensauvagement imaginaire du roman, d'une partie de son architecture cognitive et symbolique. La subtilité anthropologique du texte et de sa poétique/politique ${ }^{31}$ exige évidemment du lecteur qu'il s'aventure lui aussi à découvert dans l'univers complexe et nécessaire des signes du destin :

C'était une ferme de bonne apparence [...]. La bergerie était longue, la grange était haute, à murs lisses comme la main. Il y avait sous le hangar deux grandes charrettes et quatre charrues, avec leurs fouets, leurs colliers, leurs équipages complets, dont les toisons de laine bleue se salissaient à la poussière fine qui tombait des greniers [...].

Il aimait à se voir arriver dans la cour, à sentir contre son épaule la barrière qui 
tournait, et le coq qui chantait sur le mur [...]. Il aimait la grange et les écuries ; [...] il aimait les petits sabots de mademoiselle Emma sur les dalles lavées de la cuisine [...].

Elle le reconduisait toujours jusqu'à la première marche du perron. Lorsqu'on n'avait pas encore amené son cheval, elle restait là. On s'était dit adieu, on ne parlait plus [...]. Une fois, par un temps de dégel, l'écorce des arbres suintait dans la cour, la neige sur les couvertures des bâtiments se fondait. Elle était sur le seuil [...]. (1971, 15-19).

Un jour, elle [Emma] tira de son sac six petites cuillers en vermeil (c'était le cadeau de noces du père Rouault), en le priant d'aller immédiatement porter cela, pour elle, au mont-de-piété ; et Léon obéit, bien que cette démarche lui déplût. Il avait peur de se compromettre. (Flaubert, 1971, 295).

\section{BIBLIOGRAPHIE}

BOPP, Léon, 1951, Commentaire sur Madame Bovary, Neuchâtel, La Baconnière.

BORGES, Jorge Luis, 1986, L'art narratif et la magie, Discussion, Paris, Gallimard, p. 90-100.

BORNAND, Sandra et LEGUY, Cécile,2013, Pour une anthropologie pragmatique et énonciative, Anthropologie des pratiques langagières, Paris, A. Colin, p. 147-167.

BOURDIEU, Pierre, 2000, la Dialectique du défi et de la riposte, Esquisse d'une théorie de la pratique,

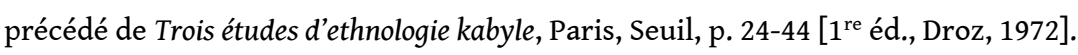

BOSQUET, Amélie, 1978, la Normandie romanesque et merveilleuse : légendes et superstitions populaires de cette province, Brionne, Gérard Monfort [1 $1^{\mathrm{re}}$ éd., Paris, Techener, 1845].

BOUTEILLER, Marcelle, 2010, Sorciers et jeteurs de sorts, s.l., Alice Lyner, [préface de C. LéviStrauss]

[1 $1{ }^{\text {re }}$ éd., Paris, Plon, 1958].

DISEGNI, Silvia, 1995, Envoûtement dans deux romans normands du XIX siècle : Madame Bovary et

l'Ensorcelée, Annali dell'Istituto universitario orientale, Sezione Romana, XXXVII, nº 1, p. 6-50.

DU CAMP, Maxime, 1906, Souvenirs littéraires, I, Paris, Hachette.

FABRE, Daniel, 2010, D'une ethnologie romantique, Savoirs romantiques : une naissance de l'ethnologie, in Daniel Fabre et Jean-Marie Privat (éds), Nancy, P.U.N., coll. « EthnocritiqueS », p. 5-75.

FAVRET-SAADA, Jeanne, 1971, le Malheur biologique et sa Répétition, Annales E.S.C., nº 3-4, p. 873-874.

FAVRET-SAADA, Jeanne, 1977, les Mots, la mort, les sorts, Paris, Gallimard.

FAVRET-SAADA, Jeanne, 2009, la Force anormale, Désorceler, s.l., Édition de l’Olivier, p. 69-77.

FLAUBERT, Gustave, 1971, Madame Bovary : mœurs de province, Édition Claudine Gothot-Mersch, Paris, Garnier Frères [ $1^{\text {re }}$ éd., Paris, 1857].

FLAUBERT, Gustave, 1973,Lettre à Ernest Chevalier,23 août 1832, Correspondance, I, Édition

Jean Bruneau, Paris, Gallimard, p. 9-10. 
FLAUBERT, Gustave, 1980,Lettre à Louise Collet, 26 août 1853, Correspondance, II, Édition Jean Bruneau, Paris, Gallimard, p. 420.

FLAUBERT, Gustave, 1997,Lettre à la princesse Mathilde, 3 septembre 1875, Correspondance,IV, Édition Jean Bruneau, Paris, Gallimard, p. 58.

FLAUBERT, Gustave, 1989, Voyage en Bretagne : par les champs et par les grèves, Bruxelles, Éditions

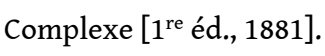

FLAUBERT, Gustave, 2001, les Mémoires d'un fou. Novembre. Pyrénées-Corse. Voyage en Italie, Édition Claudine Gothot-Mersch, Paris, Gallimard, coll. « Folio classique ».

FRANKLIN GROUT, Caroline, 1999, Heures d'autrefois. Mémoires inédits. Souvenirs intimes et autres textes, textes établis, présentés et annotés par Matthieu Desportes, Rouen, P.U.R.

FRAZER, James George, 1981, Contrôle magique du vent, le Rameau d'or, I, Paris, Laffont, coll. «Bouquins », p. 202-209 [1 ${ }^{\text {re }}$ éd., 1935].

GENETTE, Gérard, 1983, Nouveau discours du récit, Paris, Seuil.

GINZBURG, Carlo, 2010, Racines d'un paradigme indiciaire, Mythes, emblèmes, traces, Lagrasse,

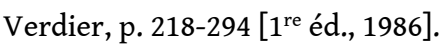

GREIMAS, Algirdas Julien, 1983, le Savoir et le Croire : un seul univers cognitif, Du Sens, ii. Essais sémiotiques, Paris, Seuil, p. 115-133.

GRIGNON, Claude et PASSERON, Jean-Claude, 1989, le Savant et le Populaire : misérabilisme et populisme en sociologie et en littérature, Paris, Hautes Études, Gallimard - Le Seuil.

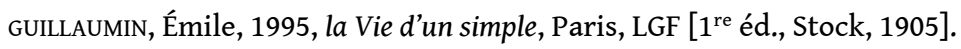

JAULIN, Robert, 1971, la Chasse gardée, la Mort sara : l'ordre de la vie ou la pensée de la mort au Tchad, Paris, U.G.E., coll. « 10/18», p. 238-240.

KLIEBENSTEIN, Georges, 2004, les Systèmes d'annonce déclarés, Figures du destin stendhalien,Paris, Presses de la Sorbonne Nouvelle, p. 87-196.

LAROUSSE, Pierre, 1877, Grand dictionnaire universel du XIX ${ }^{e}$ siècle, t. XIV, Paris.

LE BRAZ, Anatole, 1994, Magies de la Bretagne, Paris, Laffont, coll. «Bouquins » [1 ${ }^{\text {re }}$ éd., 1893, la Légende de la mort chez les Bretons armoricains, Paris, Champion].

LECERCLE, Jean-Claude, 1996, la Violence matérielle du langage : la langue et le corps, la Violence du langage, Paris, PUF, p. 237-250.

LECOEUR, Jules, 1976, Esquisses du bocage normand, II, Brionne, G. Monfort [1 $1^{\text {re }}$ éd., 1883-1887, Condésur-Noiraut, L. Morel].

MALINOWSKI, Bronislaw, 1974, Théorie ethnographique du mot magique, les Jardins de corail, Paris, F. Maspero, p. 242-244.

MAUSS, Marcel, 1991,Essai sur le don. Formes et raisons de l'échange dans les sociétés archaïques,

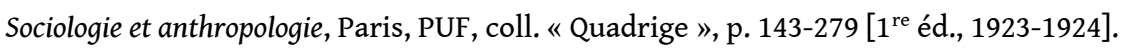

MERLEAU-PONTY, 2003, De Mauss à Claude LéviStrauss, Signes, Paris, Gallimard, p. 184-202 [1 $1^{\text {re éd., }}$ 1960].

MICHELET, Jules, 1974, le Peuple, Édition Paul Viallaneix, Paris, Flammarion [1 ${ }^{\text {re }}$ éd., 1846].

NERVAL, Gérard de, 1993, Promenades et souvenirs (1854-1855), Cuvres complètes, III, Paris, Gallimard. 
PITT-RIVERS, Julian, 1997, Anthropologie de l'honneur, Paris, Hachette/Pluriel.

SÉBILLOT, Paul, 1968, Consultations et présages, le Folklore de France : la faune et la flore, III, Paris, Maisonneuve et Larose, p. 321-326 [1 $1^{\text {re }}$ éd., Paris, Guilmoto, 1906].

\section{ANNEXES}

\section{Annexe 1}

La Belle au bois dormant

M. Mauss $(1991,253)$ observe que « ce thème de l'invitation, de la malédiction des gens non invités, de la bénédiction et de la générosité des invités, surtout quand ils sont des fées [...], est familier encore à toutes nos mœurs, à tous nos contes, toutes nos légendes. »C'est le cas, on s'en souvient, lors du baptême de la Belle au bois dormant : « une vieille fée que l'on croyait morte, ou enchantée » n'avait point été priée au « grand festin pour les Fées » marraines. Le roi lui fit donner un couvert, « mais il n'y eut pas moyen de lui donner un étui d'or massif, comme aux autres [...]. La vieille crut qu'on la méprisait, et grommela quelques menaces entre ses dents [...]. La princesse se percerait la main d'un fuseau, et en mourrait ». Le récit de Flaubert est une sorte de parodique (ou tragique) conte à l'envers puisque c'est l'héroïne elle-même qui - à terme - refuse le don nuptial :

\section{Annexe 2}

L'Évangile de Luc (14, 8-11)

Luc l'évangéliste expose une morale du don et de l'hospitalité sous forme de la parabole suivante (que le père Rouault dédaigne manifestement) : «14.8 Lorsque tu seras invité par quelqu'un à des noces, ne te mets pas à la première place, de peur qu'il n'y ait parmi les invités une personne plus considérable que toi, 14.9 et que celui qui vous a invités l'un et l'autre ne vienne te dire : Cède la place à cette personne-là. Tu aurais alors la honte d'aller occuper la dernière place. 14.10 Mais, lorsque tu seras invité, va te mettre à la dernière place, afin que, quand celui qui t'a invité viendra, il te dise : Mon ami, monte plus haut. Alors cela te fera honneur devant tous ceux qui seront à table avec toi. 14.11 Car quiconque s'élève sera abaissé, et quiconque s'abaisse sera élevé. »

\section{Annexe 3}

Robert Jaulin, « la Chasse gardée », la Mort sara : l'ordre de la vie ou la pensée de la mort au Tchad, Paris, U.G.E., « coll. 10/18 », 1971, p. 238-240.

« Nous avions coutume de bavarder le soir autour d'un petit feu [...]. J'étais arrivé depuis peu lorsque le fossoyeur du village, qui était aussi devin, dressa dans le sable un tableau géomantique à la demande du chef de terre, soucieux de savoir si la chance allait me sourire à la chasse [...]. Le gara répondit par la négative. Trois jours plus tôt en effet, un Européen était venu chasser en terre mara; il avait demandé un guide au chef de terre. Il tua deux antilopes-cheval et un katembourou. Le tout fut rapporté à son campement par les villageois, auxquels il donna une partie de la viande, le reste étant pour le guide et le chef de terre. Mais les jeunes gens du village firent main basse sur la presque totalité du gibier et Ngabra, chef de canton, n'eut qu'un petit gigot que lui 
apporta le guide; de même Naglaba, un vieillard aveugle à qui l'âge et l'appartenance à une certaine lignée conféraient un pouvoir sur la terre, fut totalement oublié.

Ils n'en montrèrent rien, mais tous deux furent très fâchés et, comme la générosité de la terre clanique et, partant, la chance à la chasse dépendaient d'eux, ils se vengèrent occultement. Ngabra et Naglaba, chacun de leur côté, touchèrent la terre de leurs doigts et dirent : « Cette terre est nôtre, et nous ne voulons plus qu'un étranger y tue de la viande. » La terre les écouta (...). Mais tout cela était loin maintenant, en partie oublié [...]. Le vieil aveugle accepta de « lever sa parole.»

\section{Document 1}

En Bretagne, durant quelques semaines, «à la pluie et au soleil », les deux marcheurs vont sac au dos, souliers ferrés aux pieds, gourdin en main, fumée aux lèvres et fantaisie en tête, « courir les champs pour coucher dans les auberges [...], pour écouter les oiseaux sous les arbres quand il a plu, et pour voir, le dimanche, les paysannes sous le porche de l'église sortir de la messe avec leurs grands bonnets blancs et leurs gros jupons rouges [...].».

Gustave et Maxime s'intéressent par exemple à « la statue de la Bonne Vierge du Crée-lait qui avait la vertu d'apporter du lait aux nourrices (on y apportait du beurre et d'autres offrandes rustiques) »; à l'enterrement rustique et pieux d'un marin «perdu à la mer »; à la place du marché du village où l'on entend sonner « les rauques syllabes celtiques mêlées au grognement des animaux et au claquement des charrettes, mais [...] ni rire dans les groupes ni bavardages sur le seuil des cabarets "; à la jeune fille nupieds riant aux éclats, etc.

Le jeune Flaubert médite sur les logiques acculturatives et les résistances culturelles indigènes, non sans (amer) réalisme ethnographique :

«Ce qu'il y a encore de plus breton dans les villes, ce sont les pauvres filles qu'on fait venir pour servir comme domestiques. Confinées dans leur service, avec qui communiqueraient-elles pour perdre le caractère natal ? Voyez-les s'arrêter dans la rue avec l'homme qui apporte chaque semaine de la campagne les œufs et le beurre. Que leur dit-il ? Il leur parle de leur village, de leurs parents ; leur frère leur envoie pour cadeau de noces une belle paire de boucles d'argent, il faudra bien les porter ; il y aura bientôt un pardon, il faudra y venir. Elles y iront donc et s'y retremperont à tout ce que la patrie a de plus distinctif, le langage et le costume ; aussi, quand elles seront de retour chez leurs maîtres, leur cœur restera là-bas, et elles en causeront ensemble en se promenant comme elles font, par bandes de dix ou vingt, sur les places et à l'entrée de la grande route, le dimanche après les vêpres.

Ainsi se conserve au milieu d'une population déjà bâtarde ce petit peuple entêté, qui tournoie dans l'autre sans y perdre ses angles. » (Flaubert, Voyage en Bretagne. Par les champs et par les grèves, 1989)

\section{Document 2}

Émile Guillaumin, la Vie d'un simple (1905), chapitre 23

Les coqs à l'engrais chantèrent un soir de décembre qu'il y avait de la neige et qu'il gelait ferme. C'était en fin de veillée, vers neuf heures; nous nous préparions à user les draps.

- Qu'est-ce qu'ils veulent annoncer, ces sales bêtes ? fit Victoire tout de suite inquiète. Signe de malheur en effet que d'entendre chanter les coqs à partir du coucher du soleil 
et jusqu'à minuit, - période du repos et du silence.

Cette infraction à la règle aurait dû cependant nous sembler naturelle de la part de ces pauvres poulets à l'engrais qui, ne sortant jamais d'un réduit enténébré, perdaient peu à peu le sentiment des heures. Mais nous étions troublés - pour avoir vu, enfants, se troubler nos proches en pareille occurrence. D'ailleurs, dans le grand calme de la nuit d'hiver, ces cocoricos avaient quelque chose de lugubre - d'autant plus qu'ils se multiplièrent : le coq des Viradon répondit aux nôtres, puis d'autres des chaumières proches et des fermes lointaines. Ce fut pendant une demi-heure un concert de modulations aiguës, comme aux heures qui précèdent l'aube.

La sérénade terminée. Victoire donna le sein à notre petit troisième qui avait juste deux mois.

Mais elle n'était guère rassurée et, bien que se défendant d'avoir peur, elle tremblait encore quand elle se mit au lit. Nous eûmes, cette nuit-là, un sommeil fiévreux et il fut décidé que les malencontreux poulets seraient vendus au plus tôt.

Comme par hasard, les mois qui suivirent, toutes sortes de malheurs nous vinrent frapper. En prenant de l'âge, je me suis libéré d'une bonne partie des croyances superstitieuses de ma jeunesse ; mais à cause de cela (sic), j'ai toujours conservé la crainte des coqs qui chantent après le coucher du soleil.

J'avais, dans un coin de mon étable, une réserve de pommes de terre. La meilleure de mes deux vaches s'étant détachée une nuit, avala goulûment un gros tubercule et s'étrangla. Je la découvris, le matin, étendue sur le dos, ballonnée, râlante. Un boucher, prévenu, m'en offrit trente francs ; je comptais la vendre trois cents francs à la fin de l'hiver...

Il me souvient que ma femme voulait acheter des habits pour notre petit Jean, et pour moi un pantalon de droguet, une casquette, une blouse. Mais on dut repousser à des temps meilleurs ces dépenses anormales. Au surplus il nous creva peu après un cochon qui pesait cent cinquante livres. Et nous eûmes des ennuis de la vache achetée en remplacement de notre pauvre étranglée.

À cause des enfants. Victoire avait cessé tout à fait de porter le lait en ville et s'était mise à faire du beurre. Or, il n'y avait pas moyen de transformer en beurre la crème qui provenait de cette nouvelle vache. Nous passions des heures et des heures à la remuer dans la baratte ou beurrier; nous avions les bras moulus de faire monter et descendre le batillon : rien! Il m'arriva un soir de le manœuvrer sans interruption de six heures à minuit ; je parvins à prendre une suée terrible, à défoncer à demi la baratte, mais non à faire du beurre...

Le père Viradon, le lendemain, m'assura que c'était un sort. Pareille mésaventure lui étant advenue dans sa jeunesse, un défaiseux de sorts lui avait donné les conseils suivants :

"- Se rendre un peu avant minuit au carrefour de la place de l'Église et poser là un petit pot neuf de six sous plein de cette mauvaise crème ; tourner douze fois autour de ce pot quand sonneraient les douze coups de minuit, en traînant au bout d'une corde de six pieds de long les chaînes d'attache des vaches ; au douzième tour, s'arrêter net, faire quatre fois le signe de la croix dans quatre directions opposées et partir au grand galop, abandonnant le pot et rapportant les chaînes.

"- Couper à chaque bête un bouquet de poils de l'oreille, un du garrot, un de la queue, les tremper dans l'abreuvoir tous les jours de la semaine sainte avant le lever du soleil, les porter à la messe le jour de Pâques et les faire brûler dans la cheminée sans être vu... 
- J'ai fait cela et la réussite a été complète, conclut Viradon. Mais le défaiseux a dû agir de son côté.

Le fou rire me prit, malgré mes embêtements, en écoutant le bonhomme raconter d'un air convaincu les détails bizarres de la cérémonie. Il me semblait le voir dans la nuit tourner autour de son pot et entendre la fretintaille de ses chaînes !

Le défaiseux était mort ; mais il avait laissé à son fils le secret de son talent, et le vieux voisin me conseillait d'avoir recours à lui. Je n'en fis rien cependant, n'ayant pas foi en ces stupidités.

Mais la bourgeoise alla conter nos peines au curé. Il vint le lendemain, aspergea l'étable avec de l'eau bénite et nous dit de n'avoir nulle crainte des sorciers.

- Ça tient tout simplement à ce que votre vache a du lait de mauvaise qualité et à ce qu'elle est dans un état de gestation avancée ; améliorez sa nourriture, donnez-lui chaque jour un peu de sel dans une ration de farineux et vous verrez que ça ira mieux. Grâce à ces bons avis, il nous devint possible de faire du beurre qui s'améliora tout naturellement quand, à la belle saison, nos vaches fraîches vélières furent pâturer sur les Craux. Si l'on se rendait bien compte de tout, on n'aurait pas souvent l'occasion de croire aux sorts.

Vers la fin de l'hiver, nous eûmes une alerte plus grave encore ; et cette fois-ci, il fallut bien, en désespoir de cause, aller trouver un rebouteux.

Notre petit Charles fut pris soudain d'un mal de gorge à caractère grave ; il refusait de prendre le sein ; sa respiration devint rauque, puis râlante. Victoire le porta d'abord à la sage-femme, puis au médecin, et ça n'avait pas l'air d'aller mieux, au contraire. Or, il y avait sur le chemin d'Agonges un homme qui « barrait » les maux de gorge d'enfants ; on venait le trouver de toutes les communes du canton et même d'ailleurs ; il sauvait, disait-on, les bébés désespérés par les docteurs. Au cours d'une veillée, l'état du petit parut tellement s'aggraver que nous décidâmes de le lui porter séance tenante. Sa mère l'emmitoufla dans un vieux châle au creux d'un oreiller et je le pris ainsi sur mon bras ; elle suivait en pleurant. Nos pas résonnaient dans le silence nocturne sur les chemins durcis par le grand gel. Triste promenade !

Nous eûmes enfin la satisfaction de frapper à la porte du guérisseur qui vint ouvrir après un moment, en caleçon et bonnet de coton. C'était un petit homme déjà âgé, à cheveux grisonnants et figure ingrate. Il marmonna des prières en faisant des signes sur le corps de notre enfant, oignit son cou d'une sorte de pommade grise et lui souffla dans la bouche par trois fois. Un chaleil fumeux éclairait cette scène étrange. J'étais impressionné; Victoire pleurait toujours silencieusement. Après qu'il eut fini, l'homme nous rassura :

- Il ira mieux demain ; mais, par exemple, il était temps de l'apporter, vous savez... Dès qu'il sera débarrassé, pour hâter sa guérison, vous irez faire brûler un cierge devant l'autel de la Sainte Vierge.

À notre demande de paiement, il répondit :

- Je ne prends rien aux pauvres gens... Mais voici un tronc où chacun met ce qu'il veut. Il désignait sur la cheminée une petite boîte carrée au couvercle percé d'une fente ; j'y glissai vingt sous et nous repartîmes en hâte, inquiets des deux aînés que nous avions laissés dormant dans la maison fermée.

Le guérisseur ne nous avait pas trompés. Vers le matin, le bébé vomit des matières aqueuses qui ressemblaient à des crachats durcis et, tout de suite soulagé, il prit le sein. Deux jours plus tard, il n'y paraissait plus.

Je me suis souvent demandé, sans pouvoir répondre ni dans un sens ni dans l'autre, si 
cette guérison fut d'effet naturel ou si les simagrées du vieux y furent pour quelque chose. Je sais que nombre de gens, très sceptiques, très fortes têtes, ne craignent pas encore aujourd'hui d'avoir recours à ces guérisseurs campagnards pour se faire barrer les dents, ou se faire dire la prière à l'occasion d'une entorse ou d'une foulure. Et d'aucuns prétendent qu'ils en ont du soulagement.

Ceci étant, un pauvre homme tout simple a bien le droit de rester perplexe, également éloigné de ceux qui affirment et de ceux qui se moquent. J'en suis encore là. »

\section{Document 3}

Madame Bovary

III. chap. 9-10 : Arrivée du père Rouault et obsèques religieuses. - brouillons, vol. 6, folio 281 / http://flaubert.univ-rouen.fr/manuscrits

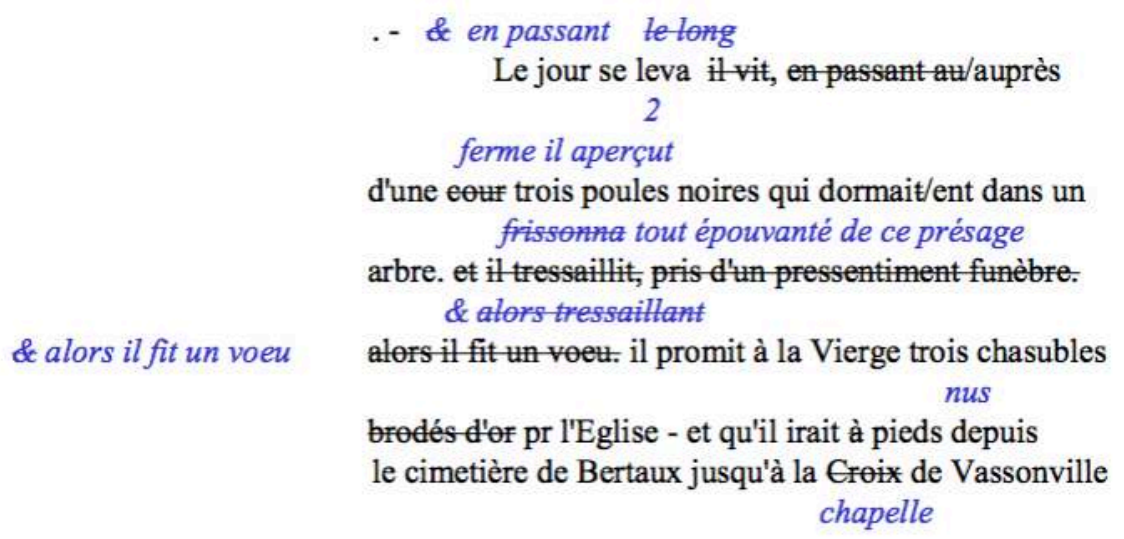

III. chap.9-10 : Arrivée du père Rouault et obsèques religieuses. - brouillons, vol.6, folio $284 \mathrm{v}$ [Page entièrement biffée] http://flaubert.univ-rouen.fr

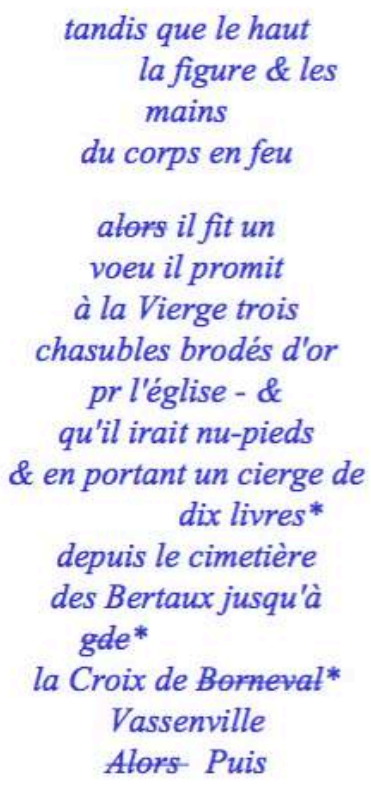

$$
\text { avait grelotta }
$$$$
\text { Le jour se leva. il eut froid. - et il regarda }
$$$$
\text { comme s'il eût marché }
$$

plusieurs fois à ses pieds croyant qu'il marchait dans l'eau jusqu'au ventre.

$$
\begin{aligned}
& \text { le long d'une cour } \\
& \text { il vit en passant dans un village trois } \\
& \text { noires }
\end{aligned}
$$

poules qui dormaient dans un arbre. - \& il frissonna tout eonfusément épouvan* d'un pressentiment funèbre 


\section{NOTES}

1. " Je dois la plupart des faits relatifs à l'enfance de mon oncle à ce que m'en a raconté la vieille bonne qui l'a élevé, morte trois ans après lui, en 1883. » (Franklin Grout, 1999, 131).

2. Citons par exemple, pour rester dans le seul domaine de la littérature de l'époque, Gérard de Nerval (Promenades et souvenirs, 1854-1855) : «Pontoise est encore une de ces villes, situées sur des hauteurs, qui me plaisent par leur aspect patriarcal, leurs promenades, leurs points de vue, et la conservation de certaines mœurs, qu'on ne rencontre plus ailleurs. On y joue encore dans les rues, on cause, on chante le soir sur le devant des portes [...]. Me direz-vous pourquoi j'aime tout le monde dans ce pays, où je retrouve des intonations connues autrefois, où les vieilles ont les traits de celles qui m'ont bercé, où les jeunes gens et les jeunes filles me rappellent les compagnons de ma première jeunesse ? Un vieillard passe: il m'a semblé voir mon grand-père; il parle, c'est presque sa voix ; - cette jeune personne a les traits de ma tante, morte à vingtcinq ans ; une plus jeune me rappelle une petite paysanne qui m'a aimé [...].» (Nerval, 1993, 688).

3. Le premier volume des Voyages pittoresques et romantiques dans l'Ancienne France de Charles Nodier, James Taylor et Alphonse de Cailleux est consacré à "l'Ancienne Normandie ». Il est publié à Paris, chez F. Didot, en 1820.

4. Dans ses Souvenirs littéraires (1906, I, 241) Maxime Du Camp décrit la Bretagne des années 1840 comme un "pays relativement éloigné et resté un peu en dehors de la civilisation par ses mœurs et par son langage ».

5. Gustave Flaubert écrivait les chapitres impairs. On pourra découvrir plus loin dans le Document 1 d'autres considérations sur la position de l'écrivain par rapport à la culture rurale (bretonne).

6. Le texte que nous proposons est un artefact qui vise à donner un accès facile et simple au travail d'écriture de Flaubert; il s'agit d'une reconstruction synthétique qui combine plusieurs essais scripturaux effectivement présents dans les brouillons de Madame Bovary. On pourra se faire une idée plus conforme à la réalité factuelle en consultant le site I, chap. 1 : Enfance de Charles - brouillons, vol. 1, folios 14 et 15 .

7. Jules Michelet $(1974,166)$ conseille aux «classes cultivées» et aux «sages» (aux lettrés, aux intellectuels dirions-nous) «d'ajourner leur étude, de bien serrer leurs livres qui leur ont servi de peu, et de s'en aller bonnement parmi les mères et les nourrices, désapprendre et oublier ». On sait que le mot folk-lore a été proposé à la communauté savante par William J. Thoms en 1846.

8. Flaubert n'a que mépris (d'artiste) pour le roman rustique et « l'assommant bruit des galoches celtiques » $(1853,420)$.

9. Gustave accompagnait parfois son père puis sa mère dans la campagne normande proche pour la collecte de leurs baux et fermages. Ses parents possédaient ainsi plusieurs propriétés dans les environs de Rouen, comme la ferme Geffosse, à Pontl'Évêque et celle du Coteau, à Deauville. Le 23 août 1832, il écrit par exemple à son très jeune ami Ernest Chevalier: "Nous avons été l'autre jour à Courtavant où il y a une ferme de papa. Nous avons pêché et comme tu sais qu'on ne peut pas pêcher (du poisson) sans eau, donc, il y avait de l'eau et une petite barque ; je me suis bien amusé et si tu y avais été, tu aurais éprouvé la même joie que moi » $(1973,9)$. Et le 3 septembre 1875, à 
la mort de sa mère, il écrira ainsi à la Princesse Mathilde: "J'ai vendu très avantageusement ma ferme de Deauville. » $(1997,978)$.

10. Lire plus loin, Document 2, sous la plume rustique d'Émile Guillaumin (1995, 132-137), le récit que lui fit son informateur, le métayer Tiennon,à propos d'une série de «malheurs » qui s'abattirent soudain sur sa famille. On verra combien dans les campagnes françaises des années 1840-1850 (en Bourbonnais ici), les régimes de créance sont incertains, et combien chez les humbles ou les «simples» la belligérance intraculturelle dans les positions d'énonciation domine.

11. Flaubert avait pu aussi lire sous la plume de son amie rouennaise Amélie Bosquet $(1978,219)$ qu'on «tire des présages heureux ou funestes de quelques animaux. Si une Araignée qui file descend sur une personne, elle lui annonce une recette prochaine d'argent ». Voir aussi sur cette mantique, entre autres, Paul Sébillot $(1968,323)$.

12. Sur « les systèmes interprétatifs du mal, du malheur et du surnaturel » auxquels les curés de campagne sont confrontés à l'époque moderne, voir Jeanne Favret-Saada (1971, 873-874).

13. Selon A. Le Braz, folkloriste breton du XIX siècle (1994, 103), «les intersignes annoncent la mort. Ils sont comme l'ombre, projetée en avant, de ce qui doit arriver [...]. La personne à qui se manifeste l'intersigne est rarement celle que la mort menace [...].» L'auteur ajoute: «Les personnes qui nient les intersignes [...] les nient uniquement parce qu'elles ne savent ni les voir, ni les entendre; peut-être aussi parce qu'elles craignent et qu'elles ne veulent rien entendre ni rien voir de l'autre vie » (Idem, 103). Le dictionnaire Littré définit, lui, sur un mode distancié et purement romanesque l'intersigne comme « le lien mystérieux que l'imagination se plaît à établir entre deux faits qui se produisent au même moment, souvent à de grandes distances l'un de l'autre, et dont l'un est considéré comme le pronostic de l'autre ». L'ethno-logique a disparu.

14. Dans les brouillons Flaubert avait d'abord songé à l'expression "pressentiment funèbre » en lieu et place de " présage », cf. Document 3.

15. Dans Madame Bovary les maisons sont des maisonnées du malheur : «- Ah ! c'est la fin pour moi (père Rouault), voyez-vous! J'ai vu partir ma femme..., mon fils après..., et voilà ma fille, aujourd'hui ! Il voulut s'en retourner tout de suite aux Bertaux, disant qu'il ne pourrait pas dormir dans cette maison-là. » $(1971,346)$ Rouault est bien " pris » dans un enchaînement de malheurs répétés et incompréhensibles (pour lui).

16. Sur les enjeux sytémiques de l'obligation de réciprocité rituelle (et de l'éventuelle transgression de la transaction symbolique), on se contentera ici de renvoyer aux hypothèses de M. Mauss $(1991,227):$ «La circulation des biens suit celle des hommes [...], des festins, des rites, des cérémonies et des danses, même celle des plaisanteries et des injures. Au fond elle est la même. Si on donne les choses et les rend, c'est parce que on se donne et se rend des 'respects' [...]. Mais aussi c'est qu'on se donne en donnant, et, si on se donne, c'est qu'on se doit - soi et son bien - aux autres. »

17. La mère du marié qui n'avait été « consultée ni sur la toilette de la bru, ni sur l'ordonnance du festin " (précisément), n'avait, elle, "pas desserré les dents de la journée »... autre forme (silencieuse cette fois) de malaise dans l'alliance qui se noue $(1971,31)$. Et Charles lui-même ne brille pas dans les jeux discursifs propres au festin de noces (un authentique potlatch) et à ses us folkloriques : «Il répondit médiocrement 
aux points, calembours, mots à double entente, compliments et gaillardises que l'on se fit un devoir de lui décocher dès le potage. » $(1971,31)$

18. « Au XIX siècle, la « force » circulait plus librement qu'aujourd'hui : il arrivait à des individus ordinaires - ni désorceleurs ni sorciers - de jeter un sort pour défendre le droit et la justice» (Favret-Saada, 2009, 69). Les folkloristes normands de l'époque attestent de la popularité de ce fonds culturel : «Ces histoires de gens qui se vengent en jetant des sorts ou en jouant des tours sont racontées tous les jours et partout dans le pays. » (Lecoeur, 1976, 49).

19. Il y a corrélation anthropologique fréquente entre la violation d'un interdit (ou même plus ordinairement l'anormalité d'un comportement) et la notion de présage ou d'augure. Pour une approche sémiotique et historique de la «schizie intraculturelle fondamendale " entre le savoir et le croire si caractéristique (apparemment) de la civilisation occidentale moderne et des logiques hybrides (en fait) du «fiduciaire » et du «logique », voir par exemple Greimas (1983, 115-133).

20. Comme le faisait remarquer Malinowski en bon pragmaticien relativiste (1974, 242-244), lorsqu'on se place du point de vue indigène d'une culture donnée, «une formule magique n'est pas une page de folklore et encore moins un document ethnographique. C'est un acte de parole qui permet de libérer telle ou telle force particulière et exerce une influence très puissante sur les phénomènes de la nature et la conduite humaine [...].» Voir aussi Lecercle (1996, 237-250). Mauss l'ethnographe (1991, 259) signale qu'en France, jusqu'à récemment encore, l'abstention d'un invité au festin du mariage était « bien mauvais signe, présage et preuve d'envie, de 'sort' ».

21. En un sens, la pensée sauvage du récit et de la régie auctoriale est en homologie avec la logique temporelle du jeteur de sort (et du lecteur pris dans les rets du texte...) qui, « poursuivant en définitive le dépérissement ou l'anéantissement total, assigne un laps de temps déterminé au développement total du maléfice » (Bouteiller, 2010, 85).

22. Dans le micro-système sémiotique du texte, le «bas bout » de la table s'oppose au « haut bout » comme le menu peuple, ancillarisé ou non, à l'aristocratie (moribonde). Ainsi, lors du bal à la Vaubyessard, «au haut bout de la table [...], courbé sur son assiette remplie, et la serviette nouée dans le dos comme un enfant, un vieillard mangeait, laissant tomber de sa bouche des gouttes de sauce [...]. C'était le vieux duc de Laverdière [...] qui avait été, disait-on, l'amant de la reine Marie-Antoinette [...]. Un domestique, derrière sa chaise, lui nommait tout haut, dans l'oreille, les plats qu'il désignait du doigt en bégayant [...]. » (Flaubert, 1971, 50)

23. Voir des exemples de conte de fées, de texte religieux et d'anthropologie exotique en Annexes 1, 2 et 3.

24. "Comme il passait par Vassonville, il aperçut, au bord d'un fossé, un jeune garçon assis sur l'herbe. - Êtes-vous le médecin? demanda l'enfant. Et, sur la réponse de Charles, il prit ses sabots à ses mains et se mit à courir devant lui »... $(1971,14)$.

25. Les dictionnaires d'autrefois définissent l'expression Mots couverts ou paroles couvertes comme des «mots ambigus » et qui "cachent un autre sens que celui qui se présente d'abord ». Sur la notion de "parole agissante », sur le concept d'agentivité et sur les «ruses des dominés pour exprimer leur position, parfois subversivement, malgré tout », voir Bornand et Leguy (2013, 147-167).

26. Sur la diabolie des éléments déchaînés (pluies diluviennes, vents fous, etc.) et leur maîtrise magique ou pas, voir par exemple Frazer (1981, 202-209). 
27. Aux dires même de mademoiselle Rouault, aux Bertaux et dans les fermes voisines, lors des grands froids, les «loups courent les champs, la nuit»... $(1971,17)$. Le syncrétisme culturel et la mémoire longue du roman laissent entendre, sotto voce, combien l'homme des champs qui adorerait volontiers le Veau d'or s'éprouve aussi comme l'héritier lointain du laboureur mythique, maudit de Dieu. On se souvient de la malédiction biblique : "Caïn se jeta sur son frère Abel et le tua [...]. Le Seigneur dit à Caïn : [...] La voix du sang de votre frère crie de la terre jusqu'à moi. Vous serez donc maintenant maudit sur la terre [...]. Quand vous l'aurez cultivée, elle ne vous rendra point son fruit ", Genèse (IV, 8-12). Or, le père Rouault voyait (entre autres) dans le mariage de sa fille une solution à ces problèmes d'intendance : «Il n'eût pas été fâché qu'on le débarrassât de sa fille, qui ne lui servait guère dans sa maison. Il l'excusait intérieurement, trouvant qu'elle avait trop d'esprit pour la culture, métier maudit du ciel, puisqu'on n'y voyait jamais de millionnaire.» $(1971,25)$ C'est dire si le fatum pagano-antique (fût-ce par dérision ironique chez Flaubert) et les catégories mythicoreligieuses testamentaires incorporées travaillent sublimalement le récit.

28. Le sort du récit, son ressort dramatique et ses sortilèges sémiotiques si l'on peut dire, sa facture et sa fracture en somme, est fatalement déjà scellé lors de la première rencontre de l'officier de santé et de la jeune demoiselle des Bertaux (le père d'Emma s'est cassé la jambe la veille au soir) : "La fracture était simple [...]. Afin d'avoir des attelles, on alla chercher, sous la charretterie, un paquet de lattes. Charles en choisit une, la coupa en morceaux et la polit avec un éclat de vitre, tandis que la servante déchirait des draps pour faire des bandes, et que mademoiselle Emma tâchait de coudre des coussinets [...] ; tout en cousant, elle se piquait les doigts, qu'elle portait ensuite à sa bouche pour les sucer » $(1971,16)$. Il convient bien sûr d'accepter que le discours du roman lui-même soit superstitieux en quelque façon, et que l'expérience de lecture soit non pas cette fameuse immersion que semble fétichiser la narratologie contemporaine mais plutôt cette «insertion " dans "tous les systèmes dont notre culture est faite " par exposition à un "universel latéral» (et non un universel de "surplomb»), qui serait proche de l'expérience ethnographique, « incessante mise à l'épreuve de soi par l'autre et de l'autre par soi »(Merleau-Ponty, 2003, 193). La superstition du roman n'exclut pas évidemment sa subtilité savante: " Les Latins donnèrent d'abord le nom de sortes à des morceaux de bois enfilés avec un cordon, dont ils se servaient pour consulter le destin »... (Larousse, 1877, 898).

29. Ce que Jeanne Favret-Saada $(1977,253)$ dit de l'ensorcelé vaut ici pour la victime d'une malédiction coutumière : « Le 'je ' [...] c'est l'ensemble constitué par lui-même et ses possessions [...]. On ne saurait distinguer corps et biens parce que les biens font corps avec celui dont ils portent la marquent du nom. » L'incipit de la lettre le signifie aussi sobrement que clairement : «J'ai eu un malheur à ma charretterie [...].» (1971, 176).

30. Pour comprendre comment un texte littéraire peut être déculturé, il suffirait de comparer la doxa académique et le sociocentrisme naif - «Emma a demandé qu'on lui épargne les plaisanteries d'usage (...); elle se sent inadaptée à ce milieu de grosse animation paysanne [...]. Il n'en faut pas davantage pour indisposer un des invités qui s'empresse d'aller grossir un petit groupe de mécontents malicieusement croqués par l'auteur " (Bopp, 1951, 59) - avec les horizons interprétatifs ouverts par une culture vive à l'affût de l'expérience de l'improbable et de l'autre - "J'ai distingué deux processus de causalité; l'un, naturel, qui est le résultat incessant d'opérations 
incontrôlables et infinies ; l'autre, magique, lucide et limité, où les détails prophétisent. Pour le roman, je pense que la seule honnêteté possible se trouve dans le second" (Borges, 1986, 100). Les narratologues reconnaissent eux-mêmes avoir peu pris en compte «la fonction prédictive» indiquant non les causes antérieures mais bien les conséquences ultérieures de la situation diégétique, des texes bibliques aux oracles antiques, des rêves prémonitoires aux sorcières de Macbeth, etc. (Genette, 1983, 62-63).

31. Ce n'est évidemment pas un hasard sous la plume si exigeante du stylisticien Flaubert si le «bas bout de la table » et le présent de noces du cousin (« qui même avait apporté...») sont placés l'un comme l'autre entre parenthèses, marques graphiques d'une régie narrative métadiscursive qui en sait/dit long sur la conduite du temps fictionnel et sursigne ponctuatif d'un écart social (une mise à l'écart - à vrai dire) ou d'une marge culturelle (refus du rite et transgression des règles de socialité). La parenthèse à la fois isole littéralement et métaphoriquement et attire l'attention... C'est ce mode de travail du texte et cette tension intradialogique à même l'écriture qui intéressent l'ethnocritique que nous pratiquons ici. Voir .

\section{RÉSUMÉS}

Flaubert a fait l'expérience enfantine (servante rustique) puis juvénile (voyages dans la France du Sud) et jeune adulte (Bretagne) du dépaysement intraculturel. L'auteur de Madame Bovary (1857) ne s'interdit pas d'ébaucher (principalement dans ses brouillons) des fragments d'ethnographie des mours de province, mais ces effets de réel sont secondaires par rapport à la pensée sauvage du roman. Nous étudions ici comment les mauvais signes et les paroles de malheur jouent un rôle décisif dans l'efficacité symbolique d'un récit à l'imaginaire beaucoup plus hétérophonique et inouï que les lectures autorisées ne semblent le lire/dire. C'est donc vers une poétique des intersignes que nous conduit ici l'analyse ethnocritique du récit, à charge pour le lecteur d'entrer dans le processus de coopération interprétative et en dialogie ouverte avec des cosmologies aussi familières en fait que souvent minorées et tenues à bonne (?) distance, sans profit littéraire.

Flaubert experienced intra-cultural disorientation as a child (country maidservant), then as an adolescent (trips to the southern part of France) and as a young adult (Brittany). The author of Madame Bovary (1857) does not hesitate (mainly in his rough drafts) to produce pieces on the ethnography of provincial customs, but these effets de réel are less important than the aspects that reflect the savage mind. In this article we explore the way in which bad omens and curses that bring bad luck play a decisive role in the symbolic effectiveness of a narrative characterised by an imaginary which is much more heterophonic and "un-heard of" than the usual interpretations seem to admit. Our ethnocritical analysis thus leads us toward a poetics of premonitory signs; it is up to the reader to enter into a process of interpretative cooperation and open dialogics with cosmologies which, though familiar, are often underestimated and kept at a safe distance, thus losing their literary value. 
INDEX

Thèmes : ethnocritique

Keywords : Madame Bovary, Ethnocriticism, Forms of Orality, Premonitory Signs, Heterophony, Symbolic Wilding, Interpretative Cooperation

Mots-clés : Madame Bovary, intersignes, hétérophonie, ensauvagement symbolique, oralité, coopération interprétative

\section{AUTEUR}

JEAN-MARIE PRIVAT

CREM - Université de Lorraine 